01;09;10

\title{
Генерация фотоакустического сигнала двухслойными прозрачными образцами с поглощающей подложкой
}

\author{
() Т.Х. Салихов, Н. Меликхуджа, А. Махмалатиф
}

Таджикский национальный университет,

Душанбе, Таджикистан

E-mail: tsalikhov@mail.ru

Поступило в Редакцию 21 января 2019 г.

В окончательной редакции 21 января 2019 г.

Принято к публикации 15 февраля 2019 г.

Предложена теория генерации акустического сигнала в буферный газ фотоакустической камеры, когда двухслойный образец является прозрачным, а подложка поглощающей. Показано, что частотная зависимость амплитуды фотоакустического сигнала, генерируемого поглощающей подложкой, подчиняется закону $\propto \omega^{-1}$ для случая, когда длина пробега фотона меньше длины тепловой диффузии, и $\propto \omega^{-3 / 2}$ для обратного случая.

DOI: 10.21883/PJTF.2019.09.47710.17707

Известно [1-4], что метод фотоакустической (ФА) спектроскопии широко используется для исследования кинетических, оптических, акустических и теплофизических свойств различного рода жидких и твердотельных образцов, в том числе неоднородных, гиротропных и наносистем [5-11]. В [12] было показано, что нелинейные составляющие ФА-сигнала, детектируемые микрофоном, позволяют определить температурную зависимость теплофизических и оптических величин исследуемых образцов. В зависимости от геометрии эксперимента существуют различные варианты линейной теории ФА-эффекта в двух- и многослойных твердотельных системах. Наиболее распространенной является теория Фужи, Моритани и Накай [13], которая является развитием одномерной теории Розенсвайга-Гершо [14], построенной для однослойных образцов. Однако в [13], как и в [14], рассматривался случай, когда подложка является прозрачной. В [15] предложена теория генерации ФА-сигнала для однослойных прозрачных систем, находящихся на поглощающей подложке. Целю настоящей работы является построение теории генерации ФА-сигнала прозрачными двухслойными системами, находящимися на поглощающей подложке.

Исходим из следующей системы линейных уравнений теплопроводности для газового слоя $(g)$, первого $(s 1)$ и второго $(s 2)$ слоев образца и подложки $(b)$ :

$$
\begin{gathered}
C_{p g} \frac{\partial T_{g}}{\partial t}=\kappa_{g} \frac{\partial^{2} T_{g}}{\partial x^{2}}, \quad 0 \leq x \leq l_{g} ; \\
C_{p 1} \frac{\partial T_{s 1}}{\partial t}=\kappa_{1} \frac{\partial^{2} T_{s 1}}{\partial x^{2}}, \quad-l_{1} \leq x \leq 0 ; \\
C_{p 2} \frac{\partial T_{s 2}}{\partial t}=\kappa_{2} \frac{\partial^{2} T_{s 2}}{\partial x^{2}}, \quad-\left(l_{1}+l_{2}\right) \leq x \leq-l_{1} ; \\
C_{p b} \frac{\partial T_{b}}{\partial t}=\kappa_{b} \frac{\partial^{2} T_{b}}{\partial x^{2}}+\frac{\beta_{b} A_{b} I_{2}}{2} e^{\beta_{b}\left\langle x+l_{1}+l_{2}\right\rangle}\left(1+e^{i \omega t}\right), \\
-\left(l_{b}+l_{1}+l_{2}\right) \leq x \leq-\left(l_{1}+l_{2}\right),
\end{gathered}
$$

где $I_{0}-$ интенсивность падающего луча; $\omega-$ частота ее модуляции; $I_{1}=I_{0}\left(1-R_{s 1}\right), I_{2}=I_{1}\left(1-R_{s 2}\right) ; C_{p i}, \kappa_{i}$, $R_{i}$ - теплоемкости единицы объема, коэффициенты теплопроводности и отражения соответствующих слоев; $\beta_{b}, A_{b}$ - коэффициент поглощения и поглощательная способность (степень черноты) подложки; $l_{g}, l_{1}, l_{2}, l_{b}-$ толщины газового слоя, первого и второго слоев образца и подложки соответственно.

Возмущения температур $T_{i}^{\prime}(x, t)$ представим в виде $T_{i}^{\prime}(x, t)=T_{0 i}(x)+\Phi_{i}(x, t)$, где $T_{0 i}(x)$ и $\Phi_{i}(x, t)-$ соответственно равновесная и колебательная части возмущения. Далее, представив изменение величины $\Phi_{i}(x, t)$ по времени в виде $\Phi(x, t)=\Phi(x, \omega) \exp (i \omega t)$, из системы уравнений (1)-(4) для нее получим

$$
\begin{gathered}
\frac{\partial^{2} \Phi_{g}}{\partial x^{2}}=-\sigma_{g}^{2} \Phi_{g}=0, \quad 0 \leq x \leq l_{g}, \\
\frac{\partial^{2} \Phi_{s 1}}{\partial x^{2}}-\sigma_{s 1}^{2} \Phi_{s 1}=0, \quad-l_{1} \leq x \leq 0, \\
\frac{\partial^{2} \Phi_{s 2}}{\partial x^{2}}-\sigma_{s 2}^{2} \Phi_{s 2}=0, \quad-\left(l_{1}+l_{2}\right) \leq x \leq-l_{1}, \\
\frac{\partial^{2} \Phi_{b}}{\partial x^{2}}-\sigma_{b}^{2} \Phi_{b}=-\frac{\beta_{b} A_{b} I_{2} e^{\beta_{b}\left(x+l_{1}+l_{2}\right)}}{2 \kappa_{b}}, \\
-\left(l_{b}+l_{1}+l_{2}\right) \leq x \leq-\left(l_{1}+l_{2}\right),
\end{gathered}
$$

где $\sigma_{i}^{2}=i \omega / \chi_{i}, \sigma_{i}=(1+i) a_{i}, a_{i}=1 / \mu_{i}, \mu_{i}=\left(2 \chi_{i} / \omega\right)^{1 / 2}-$ длина тепловой диффузии, $\chi_{i}=\kappa_{i} / C_{p i}-$ температуропроводности соответствующих слоев.

Решения однородных уравнений $(5)-(7)$ и неоднородного (8) имеют следующий вид:

$$
\begin{gathered}
\Phi_{g}(x, \omega)=\Theta e^{-\sigma_{g} x}, \quad \Phi_{s 1}(x, \omega)=\gamma_{1} e^{\sigma_{s 1} x}+\gamma_{2} e^{-\sigma_{s 1} x}, \\
\Phi_{s 2}(x, \omega)=G_{1} e^{\sigma_{s 2}\left(x+l_{1}\right)}+G_{2} e^{-\sigma_{s 2}\left(x+l_{1}\right)}, \\
\Phi_{b}(x, \omega)=U e^{\sigma_{b}\left(x+l_{1}+l_{2}\right)}+E_{b} e^{\beta_{b}\left(l_{1}+l_{2}+x\right)},
\end{gathered}
$$

где

$$
E_{b}=\frac{\left(1-R_{s 1}\right)\left(1-R_{s 2}\right) I_{0} A_{b} \beta_{b}}{2 \kappa_{b}\left(\sigma_{b}^{2}-\beta_{b}^{2}\right)}
$$


а величины $\Theta, \gamma_{1}, \gamma_{2}, G_{1}, G_{2}$ и $U$ являются комплексными амплитудами колебания температур в соответствующих слоях. Шесть граничных условий

$$
\begin{gathered}
\Phi_{g}(0, \omega)=\Phi_{s 1}(0, \omega), \Phi_{s 1}\left(-l_{1}, \omega\right)=\Phi_{s 2}\left(-l_{1}, \omega\right), \\
\Phi_{s 2}\left(-l_{2}-l_{1}, \omega\right)=\Phi_{b}\left(-l_{2}-l_{1}, \omega\right) \\
\left.\kappa_{g} \frac{\partial \Phi_{g}}{\partial x}\right|_{x=0}=\left.\kappa_{1} \frac{\partial \Phi_{s 1}}{\partial x}\right|_{x=0},\left.\kappa_{1} \frac{\partial \Phi_{s 1}}{\partial x}\right|_{x=-l_{1}}=\left.\kappa_{2} \frac{\partial \Phi_{s 2}}{\partial x}\right|_{x=-l_{1}}, \\
\left.\kappa_{2} \frac{\partial \Phi_{s 2}}{\partial x}\right|_{x=-l_{1}-l_{2}}=\left.\kappa_{b} \frac{\partial \Phi_{b}}{\partial x}\right|_{x=-l_{1}-l_{2}}
\end{gathered}
$$

позволяют получить систему линейных алгебраических уравнений для определения величин $\Theta, \gamma_{1}, \gamma_{2}, G_{1}, G_{2}, U$. Решая эту систему и принимая во внимание условия малости $g=\left(\kappa_{g} \sigma_{g} / \kappa_{1} \sigma_{s 1}\right) \ll 1$, для колебания температуры в газовом слое получим следующее выражение:

$$
\begin{aligned}
& \Theta=4 s b E_{b}\left(1-r_{b}\right) /\left\{\left[(1-s) e^{\sigma_{s 1} l_{1}}-(1+s) e^{-\sigma_{s 1} l_{1}}\right]\right. \\
& \left.\times(1-b) e^{-\sigma_{s 2} l_{2}}+\left[(1+s) e^{\sigma_{s 1} l_{1}}-(1-s) e^{-\sigma_{s 1} l_{1}}\right](1+b) e^{\sigma_{s 2} l_{2}}\right\},
\end{aligned}
$$

где $s=\left(\kappa_{2} \sigma_{s 2} / \kappa_{1} \sigma_{s 1}\right), b=\left(\kappa_{b} \sigma_{b} / \kappa_{2} \sigma_{s 2}\right), r_{b}=\beta_{b} / \sigma_{b}$.

Акустическое колебание давления в газовом слое определяется усреднением $\Phi_{g}(x, \omega)$ по длине тепловой диффузии в газе и описывается выражением [13,14]:

$$
\delta p(\omega)=\frac{\gamma p_{0} \Theta}{\sqrt{2} T_{0} a_{g} l_{g}} \exp [i(\omega t-\pi / 4)],
$$

где $\gamma$ - показатель адиабаты, а $p_{0}$ и $T_{0}-$ соответственно начальные значения давления и температуры в ФАкамере.

Из выражения (9) видно (и это является очевидным), что для рассматриваемого случая колебание давления в газовом слое возможно, только когда оба слоя образца являются термически тонкими, т.е. $l_{i} \ll \mu_{s i}$ и $\exp \left( \pm \sigma_{s i} l_{i}\right) \approx 1$. Тогда

$$
\begin{aligned}
& {\left[(1-s) e^{\sigma_{s 1} l_{1}}-(1+s) e^{-\sigma_{s 1} l_{1}}\right](1-b) e^{-\sigma_{s 2} l_{2}}} \\
& \quad+\left[(1+s) e^{\sigma_{s 1} l_{1}}-(1-s) e^{-\sigma_{s 1} l_{1}}\right](1+b) e^{\sigma_{s 2} l_{2}} \approx 4 b s .
\end{aligned}
$$

С другой стороны, подложка характеризуется двумя характерными длинами: $l_{\beta}=\beta_{b}^{-1}$ и $\mu_{b}$ - длинами пробега фотона и тепловой диффузии. В зависимости от соотношения между этими величинами могут реализоваться два различных случая.

1. При условии $l_{\beta} \ll \mu_{b}$ получим

$$
\delta p_{1}(\omega)=\frac{Y \eta(1-i)}{4 a_{g}}\left(\frac{\mu_{b}}{\kappa_{b}}\right) \exp [i(\omega t-\pi / 4)],
$$

где $Y=\gamma p_{0} I_{0} / 2 \sqrt{2} l_{g} T_{0}, \eta=\left(1-R_{s 1}\right)\left(1-R_{s 2}\right) A_{b}$. Нетрудно заметить, что в этом случае частотная зависимость амплитуды генерируемого звука подчиняется закону $\propto \omega^{-1}$.

2. Пусть $l_{\beta} \gg \mu_{b}$, тогда справедливо выражение

$$
\delta p_{2}(\omega)=\frac{Y \eta \beta_{b} \mu_{b}}{4 a_{g}}\left(\frac{\mu_{b}}{\kappa_{b}}\right) \exp [i(\omega t-3 \pi / 4)],
$$

из которого следует, что спад амплитуды возбуждаемого ФА-сигнала с ростом частоты подчиняется закону $\propto \omega^{-3 / 2}$.

Таким образом, в работе предложена теория генерации ФА-сигнала двухслойными прозрачными твердотельными образцами, находящимися на поглощающей подложке. Установлено, что вклад от поглощающей подложки в генерируемый ФА-сигнал имеет место лишь в том случае, когда оба слоя образца являются термически тонкими.

\section{Список литературы}

[1] Гусев В.Э., Карабутов А.А. Лазерная оптоакустика. М: Наука, 1991. 304 c.

[2] Егерев С.В., Лямшев Л.М., Пученков О.В. // УФН. 1990. T. 60. № 9. C. 111-154.

[3] Лямшев Л.М. Лазерное термооптическое возбуждение звука. М.: Наука, 1989. 237 с.

[4] Винокуров С.А. // ЖПС. 1985. Т. 42. № 1. С. 5-16.

[5] Глазов А.Л., Морозов Н.Ф., Муратиков К.Л. // Письма в ЖТФ. 2016. Т. 42. В. 2. C. 23-29.

[6] Глазов А.Л., Калиновский В.С., Контрош Е.В., Муратиков К.Л. // Письма в ЖТФ. 2016. Т. 42. В. 11. С. 33-40.

[7] Морозов Н.Ф., Глазов А.Л., Муратиков К.Л. // ДАН. 2018. T. 479. B. 4. C. $382-385$.

[8] Митюрич Г.С., Черненок Е.В., Свиридова В.В., Сердюков А.Н. // Проблемы физики, математики, техники. 2016. T. 27. B. 2. C. $18-23$.

[9] Митюрич Г.С., Лебедева Е.В., Сердюков А.Н. // Проблемы физики, математики и техники. 2016. Т. 27. В. 4. С. 1926.

[10] Usoltseva L.O., Volkov D.S., Nedosekin D.A., Korobov M.V., Proskurnin M.A., Zharov V.P. // Photoacoustics. 2018. V. 12. P. 55-66.

[11] Егерев С.В. // Изв. РАН. Сер. физ. 2018. Т. 82. № 5. С. 532 537.

[12] Мадвалиев У., Салихов Т.Х., Шарифов Д.М. // ЖТФ. 2006. T. 76. B. 6. C. 87-97.

[13] Fujii Y., Moritani A., Nakai J. // Jpn. J. Appl. Phys. 1981. V. 20. N 2. P. 361-367.

[14] Rosencwaig A., Gersho A. // J. Appl. Phys. 1976. V. 47. N 1. P. 64-69.

[15] Barros Melo W.L., Faria R.M. // Appl. Phys. Lett. 1995. V. 67. N 26. P. 3892-3894. 